\title{
The Impact of Severity of Illness at the Community Level
}

\author{
Ronald Lagoe ${ }^{1 *}$, Louise Pernisi², Shelly Littau ${ }^{1}$ \\ ${ }^{1}$ Hospital Executive Council, Syracuse, NY, USA \\ ${ }^{2}$ Upstate University Hospital, Syracuse, NY, USA \\ Email: *Hospexcl@cnymail.com
}

Received 25 November 2015; accepted 21 December 2015; published 24 December 2015

Copyright (C) 2015 by authors and Scientific Research Publishing Inc.

This work is licensed under the Creative Commons Attribution International License (CC BY). http://creativecommons.org/licenses/by/4.0/

(c) (i) Open Access

\begin{abstract}
This study evaluated the impact of severity of illness on hospital inpatients within the metropolitan area of Syracuse, New York, during January-December 2014. It demonstrated that patients with Major and Extreme severity of illness generated a substantial majority of the excess lengths of stay and adverse outcomes during this period. These patients were associated with 77 percent of the excess days for adult medicine and 100 percent of the excess days for adult surgery. They also generated hospital readmission rates that were at least 50 percent higher than those of patients with Minor and Moderate severity of illness. They were also associated with more than 75 percent of inpatients with the most frequent post admission complications. The data suggested that these populations need to be a focus of efforts to improve hospital efficiency and outcomes.
\end{abstract}

\section{Keywords}

\section{Hospitalization, Hospital Lengths of Stay, Severity of Illness}

\section{Introduction}

In the United States, efforts to improve health care continue to focus on utilization and outcomes. Studies and professional opinions have suggested that these efforts can result in better and more efficient care [1]-[4].

Much of this interest involves hospitals because acute care is the most visible and most expensive component of the health care system. In recent years, hospitals have been a focus for specific initiatives to improve utilization and outcomes such as the Medicare programs to reduce inpatient readmissions and complications, as well as statewide initiatives to focus nursing home admissions on patients with the greatest care needs [5]-[7].

These developments in health care have focused considerable attention on the need to evaluate patients by

"Corresponding author. 
severity of illness. Because of advances in medical care and other factors, numbers of patients with multiple diagnoses have increased. Especially among the elderly, it is no longer unusual for patients to experience several chronic conditions. These factors have created challenges for health professionals involved with the identification and treatment of these individuals [8]-[11].

Looking toward the future, evaluation of severity of illness is also important as hospitals focus on the highest risk populations. Supporting this process involves the movement of lower levels of illness to ambulatory and long term care services in the community. It will also involve managing patients with high severity of illness in Accountable Care and other approaches [12].

The development of electronic health care data has produced opportunities to identify and quantify the impact of severity of illness in health care patient populations. During the 1990's, $3 \mathrm{M}^{\mathrm{TM}}$ Health Information Systems developed a system for evaluating severity of illness based on a number of factors including patient's principal diagnosis, secondary diagnoses, and age. This system made it possible to identify and study large numbers of patients according to their relative levels of illness [13]. Software developed recently has also made it possible to apply severity of illness to outcomes such as inpatient hospital readmissions and complications [14] [15].

An important context for the evaluation of health care data concerning severity of illness is the community level. This is because most health care services are provided in local communities.

Additionally, in the United States, national data are not available by severity of illness because of the absence of a national database that includes all health care payors. For this reason, it is not possible to describe the distribution of patients by severity of illness at the national level.

Although national and statewide data are important, it is at the community level that health services are used through interactions between patients and providers and interactions among providers. From this perspective, within communities, the parameters and the impact of severity of illness and other indicators can be analyzed among services that work together on a daily basis.

\section{Population}

This study evaluated the severity of illness among hospital inpatients in a single acute care system, the metropolitan area of Syracuse, New York. The City of Syracuse and Onondaga County include three hospitals, Crouse Hospital (19,919 discharges excluding well newborns, 2014), St. Joseph’s Hospital Health Center (25,532 discharges), and Upstate University Hospital (26,649 discharges). Within the local health care system, the hospitals work with a combined medical staff of 1830 physicians and 12 nursing homes. The hospitals provide acute care services to a local population of approximately 600,000 and tertiary services to a population of approximately $1,400,000$.

Historically, the Syracuse hospitals have competed and cooperated in the provision of acute care within the service area through their joint planning organization, the Hospital Executive Council. This cooperation has included the use of community wide acute care data to improve efficiency and outcomes as well as the development of multihospital services [16].

Since the 1990s, the Syracuse hospitals and the Hospital Executive Council have worked with $3 \mathrm{M}^{\mathrm{TM}}$ Health Information Services in the application of severity of illness data to a variety of health care issues. These have included reduction of hospital lengths of stay, monitoring of inpatient admissions, reduction of inpatient readmissions, and reduction of inpatient complications [17]. These efforts have included use of the All Patients Refined Diagnosis Related Group (APR DRG) severity of illness system, as well as the Potentially Preventable Readmissions and Potentially Preventable Complications software.

\section{Methods}

This study evaluated the severity of illness of hospital inpatients in the acute care system of Syracuse, New York using the All Patients Refined Diagnosis Related Group Severity of Illness System. It focused on the impact of this algorithm on hospital lengths of stay, readmissions, and complications. The All Patients Refined Severity of Illness System was developed by $3 \mathrm{M}^{\mathrm{TM}}$ Health Information Systems as an adjunct of the All Patients Refined Diagnosis Related Groups. It is based on the identification of a level of severity of illness of the principal diagnosis and each secondary diagnosis for every hospital inpatient. It includes four levels of severity for each diagnosis, Minor, Moderate, Major, and Extreme. At the patient specific level, the system also includes a summary severity of illness based on the severity levels by diagnosis, as well as the patient age and other factors. The 
formula for patient severity of illness includes clinical relative weights for each indicator [13].

The study data were provided by each of the Syracuse hospitals to the Hospital Executive Council staff as administrative data through business associate agreements. In the absence of a community wide ethics committee, the study was reviewed with staff from each of the hospitals.

The study employed the Potentially Preventable Readmissions and the Potentially Preventable Complications systems to evaluate the relationships between severity of illness and inpatient outcomes. These algorithms were developed by 3M Health Information systems for use with the APR DRGs and severity of illness [15] [18].

The analyses which comprise this study were based on simple descriptive statistics. The data analysis was based on identification and comparisons of hospital inpatient discharges by severity of illness.

In this study, the All Patients Refined System was used to identify the severity of illness in populations discharged from the Syracuse hospitals during 2014. Three indicators were used in the analysis, inpatient length of stay, inpatient readmissions, and post admission complications. The analysis focused on differences in each of these indicators by severity of illness and on the relative distributions of patients among the four severity of illness categories.

The initial component of the analysis involved inpatient lengths of stay by severity of illness for adult medicine and adult surgery, the services with the largest numbers of discharges in the Syracuse hospitals. It was based on inpatient data for the combined hospitals for January-December 2014. The analysis included numbers of discharges and lengths of stay for each service by severity of illness. It also included comparisons of these stays with those of a national sample of hospital inpatients by service and severity of illness.

The second component of the study involved inpatient Potentially Preventable Readmission rates for adult medicine and adult surgery in the Syracuse hospitals. This analysis was based on readmissions within 30 days of the previous discharge. Potentially Preventable Readmissions included all of those where a clinical relationship between the initial admission and the readmission was present. Between 2009 and 2014, these services accounted for more than 75 percent of total readmissions in the Syracuse hospitals. This analysis employed inpatient hospital data for January-December 2014 to identify hospital readmission rates for each of the hospitals and the combined total. It included comparison of these rates among severity of illness categories for each of these populations.

The third component of the analysis involved Potentially Preventable Complications for pneumonia and urinary tract infections, the diagnoses associated with the largest numbers of post admission complications in the Syracuse hospitals. The analysis was based on data for Crouse Hospital and St. Joseph's Hospital Health Center, two hospitals which account for more than 60 percent of hospital inpatients in Syracuse. They were the only hospitals for which complete data were available for January-December 2014. The analysis was based on numbers of inpatient complications by severity of illness category for patients with pneumonia and urinary tract infection as a Potentially Preventable Complication and those without each complication.

\section{Results}

The first component concerned hospital lengths of stay in Syracuse, New York by severity of illness. Relevant data for the combined Syracuse hospitals for January-December 2014 are summarized in Table 1.

These data identify numbers of hospital discharges and lengths of stay in the Syracuse hospitals by All Patient Refined Severity of Illness for the two largest acute care services, adult medicine and adult surgery. This information demonstrated that, for each service, mean hospital stays increased with higher severity of illness.

Lengths of stay for patients with Moderate severity of illness were 38.3 percent longer than those with Minor severity of illness for adult medicine and 46.3 percent longer for adult surgery. Mean stays for patients with Major severity of illness were 56.4 percent longer than those with Moderate severity of illness for adult medicine and 126.0 percent longer for adult surgery. Stays for patients with Extreme severity of illness were 88.5 percent longer than those with Major severity of illness for adult medicine and 166.3 percent longer for adult surgery.

The data in Table 1 also identified differences in the distribution of hospital inpatients in Syracuse for the two major services. For adult medicine, 50.8 of inpatients were at the highest levels of severity of illness (Major or Extreme), while for adult surgery, 26.5 percent were at these levels. Perhaps most importantly with respect to hospital lengths of stay, the data in Table 1 identified differences in hospital stays and related inpatient utilization compared with national averages adjusted for severity of illness. These comparisons were adjusted to compare 
Table 1. Adult medicine and adult surgery discharges by severity of illness, Syracuse hospitals, 2014.

\begin{tabular}{|c|c|c|c|c|c|}
\hline & \multicolumn{5}{|c|}{ Severity of Illness } \\
\hline & Minor & Moderate & Major & Extreme & Total \\
\hline \multicolumn{6}{|l|}{ Adult Medicine } \\
\hline Number of Discharges & 4451 & 11,977 & 12,835 & 4158 & 33,421 \\
\hline Mean Length of Stay & 2.77 & 3.83 & 5.99 & 11.29 & 5.45 \\
\hline Severity Adjusted National Average & 2.68 & 3.72 & 5.70 & 10.64 & 5.20 \\
\hline Length of Stay Difference & 0.09 & 0.11 & 0.29 & 0.65 & 0.25 \\
\hline Patient Days Difference & 400.59 & 1317.47 & 3722.15 & 2702.70 & 8355.25 \\
\hline \multicolumn{6}{|l|}{ Adult Surgery } \\
\hline Number of Discharges & 6562 & 8552 & 4008 & 1440 & 20,562 \\
\hline Mean Length of Stay & 2.76 & 4.04 & 9.13 & 24.31 & 6.04 \\
\hline Severity Adjusted National Average & 2.99 & 4.32 & 9.00 & 20.57 & 5.95 \\
\hline Length of Stay Difference & -0.23 & -0.28 & 0.13 & 3.74 & 0.09 \\
\hline Patient Days Difference & -1509.26 & -2394.56 & 521.04 & 5385.60 & 1850.58 \\
\hline
\end{tabular}

Source: Hospital Executive Council.

national and Syracuse populations with the same severity of illness. The results of these comparisons were quantified as unit differences between stays and utilization differences in patient days.

The results of these comparisons demonstrated that a substantial majority of the excess patient days in the Syracuse hospitals for each service were generated by patients at Major or Extreme severity of illness. For Adult Medicine, of the 8355 patient days difference from the national average, 76.9 percent were produced by patients at Major or Extreme severity of illness. For Adult Surgery, all 5906 excess patient days were generated by patients at Major or Extreme severity of illness.

The second component of the study concerned patients readmitted to the Syracuse hospitals within 30 days of their initial inpatient discharge by severity of illness. Relevant Potentially Preventable Readmissions data for adult medicine and adult surgery combined between January and December 2014 are summarized in Table 2.

The data in Table 2 demonstrated that increases in severity of illness were associated with increases in monthly hospital readmission rates in the Syracuse hospitals between January-March and July-December 2014. Between patients with Minor and Moderate severity of illness within each time period, the rates increased for all three quarters at Crouse Hospital and St. Joseph's Hospital Health Center and for two of the three quarters at Upstate University Hospital. For most of these comparisons, the rates increased by more than 50 percent. Between patients with Major and Moderate severity of illness, the rates increased at all three hospitals. Between patients with Major and Extreme severity of illness, the rates increased for all three quarters at Crouse Hospital and St. Joseph's Hospital Health Center and for two of the three quarters at Upstate University Hospital.

The readmission rates for high severity of illness patients also provided information concerning the relative sizes of theses populations. For patients with Major severity, between 10 and 13 percent of medical-surgical inpatients were readmissions. For patients with Extreme severity, between 12 and 25 percent of medical-surgical inpatients were readmissions.

In a separate analysis, the Pearson Correlations between monthly readmission rates for medical-surgical patients and those in specific severity of illness categories between January 2013 and March 2015 were identified. Among the Syracuse hospitals, correlations between these rates for patients with Minor or Moderate severity of illness and rates for all medical surgical inpatients ranged between 0.3173 and 0.5620 among the hospitals, 
Table 2. Potentially preventable readmission rates by severity of Illness, medical/surgical-all payors, Syracuse hospitals, 2014.

\begin{tabular}{|c|c|c|c|c|c|}
\hline & \multicolumn{5}{|c|}{ Severity of Illness } \\
\hline & Minor & Moderate & Major & Extreme & Total \\
\hline \multicolumn{6}{|l|}{ Crouse Hospital } \\
\hline Jan-Mar 14 & 3.19 & 4.55 & 10.98 & 25.21 & 7.96 \\
\hline Jul-Sept 14 & 4.29 & 6.55 & 10.18 & 18.05 & 7.92 \\
\hline Oct-Dec 14 & 4.11 & 6.30 & 10.39 & 14.18 & 7.59 \\
\hline \multicolumn{6}{|c|}{ St. Joseph’s Hospital Health Center } \\
\hline Jan-Mar 14 & 2.37 & 7.09 & 12.66 & 18.17 & 8.99 \\
\hline Jul-Sept 14 & 3.64 & 5.77 & 13.13 & 19.29 & 8.71 \\
\hline Oct-Dec 14 & 2.70 & 6.69 & 12.73 & 22.04 & 8.79 \\
\hline \multicolumn{6}{|c|}{ Upstate University Hospital-SUNY UMU } \\
\hline Jan-Mar 14 & 3.95 & 8.33 & 11.96 & 15.04 & 9.02 \\
\hline Jul-Sept 14 & 6.92 & 6.69 & 12.41 & 14.47 & 9.11 \\
\hline Oct-Dec 14 & 5.11 & 6.83 & 12.77 & 12.34 & 9.00 \\
\hline
\end{tabular}

Complete data for April-June unavailable because of implementation of electronic medical record systems at two of the hospitals; based on 3M Health Information Systems Potentially Preventable Readmissions algorithm applied to adult medical/surgical definitions by APR DRG for readmissions within 30 days; prepared by Hospital Executive Council.

while correlations between rates for patients with Major or Extreme severity of illness and rates for all medical surgical inpatients ranged from 0.7187 - 0.8735. These correlations demonstrated that patients with Major and Extreme severity of illness were the principal drivers of variations in readmission rates.

The third component of the study concerned patients who experienced post admission complications in the Syracuse hospitals for pneumonia and urinary tract infection, the diagnoses with the largest numbers of patients for this outcome. Data for these Potentially Preventable Complications at Crouse Hospital and St. Joseph's Hospital Health Center during 2014 are summarized in Table 3.

This information demonstrated that substantial majorities of the hospital inpatients who experienced the two complications in each of the hospitals were at Major or Extreme severity of illness. Patients at these levels of severity of illness accounted for 89.3 - 92.3 percent of all inpatients with pneumonia as a complication and 75.9 - 79.1 percent of inpatients with urinary tract infection as a complication. These data were also reflected in the relatively small numbers of patients at Minor or Moderate severity of illness with pneumonia complication (10 11) and with urinary tract infection as a complication (19 - 27). These data suggested that patients with high severity of illness were probably more susceptible to these complications, even in hospital inpatient settings.

Further comparisons addressed the potential relationships between patients with and without each of the complications evaluated in the two Syracuse hospitals. For pneumonia, 89.3 - 92.3 percent of inpatients who experienced the complication were at Major and Extreme severity of illness compared with 40.2 - 41.1 percent of those who did not. For urinary tract infection, 75.9 - 79.1 percent of inpatients who experienced the complication were at Major or Extreme severity of illness, compared with 40.4 - 41.1 percent of those who did not. High severity of illness was frequently associated with each of these complications.

During the same period, for pneumonia, 7.6 - 10.7 of inpatients in the hospitals who experienced the complication were at Minor and Moderate severity of illness, compared with 58.9 - 59.7 percent of those who did not. For urinary tract infection, 20.9 - 24.1 percent of inpatients in the hospitals who experienced the complication were at Minor and Moderate severity of illness, compared with 58.8 - 59.5 percent of those without it. 
Table 3. Medical/Surgical Potentially Preventable Complications (PPC), highest volume PPC categories, by Severity of Illness, St. Joseph’s Hospital Health Center (SJHHC) and Crouse Hospital (CH), 2014.

\begin{tabular}{|c|c|c|c|c|c|c|c|c|}
\hline & \multicolumn{4}{|c|}{ Patients at Risk } & \multicolumn{4}{|c|}{ Percent of Total } \\
\hline & \multicolumn{2}{|c|}{ With PPC } & \multicolumn{2}{|c|}{ Without PPC } & \multicolumn{2}{|c|}{ With PPC } & \multicolumn{2}{|c|}{ Without PPC } \\
\hline & SJHHC & $\mathrm{CH}$ & SJHHC & $\mathrm{CH}$ & SJHHC & $\mathrm{CH}$ & SJHHC & $\mathrm{CH}$ \\
\hline \multicolumn{9}{|c|}{$\begin{array}{l}\text { PPCs 04/05-Pneumonia and Other } \\
\text { Lung Infections }\end{array}$} \\
\hline Minor & 0 & 0 & 4228 & 2982 & 0.00 & 0.00 & 19.27 & 22.75 \\
\hline Moderate & 10 & 11 & 8706 & 4845 & 7.63 & 10.68 & 39.67 & 36.96 \\
\hline Major & 44 & 26 & 7015 & 3976 & 33.59 & 25.24 & 31.96 & 30.33 \\
\hline Extreme & 77 & 66 & 1997 & 1304 & 58.78 & 64.08 & 9.10 & 9.95 \\
\hline Total & 131 & 103 & 21,946 & 13,107 & 100.00 & 100.00 & 100.00 & 99.99 \\
\hline \multicolumn{9}{|c|}{ PPC 16-Urinary Tract Infection } \\
\hline Minor & 1 & 1 & 4227 & 2981 & 0.78 & 1.27 & 19.26 & 22.70 \\
\hline Moderate & 26 & 18 & 8690 & 4838 & 20.16 & 22.78 & 39.59 & 36.84 \\
\hline Major & 61 & 29 & 6998 & 3973 & 47.29 & 36.71 & 31.88 & 30.26 \\
\hline Extreme & 41 & 31 & 2033 & 1339 & 31.78 & 39.24 & 9.26 & 10.20 \\
\hline Total & 129 & 79 & 21,948 & 13,131 & 100.01 & 100.00 & 99.99 & 100.00 \\
\hline
\end{tabular}

Prepared by Hospital Executive Council.

\section{Discussion}

As health care evolves in the United States, the importance of evaluating the degree of illness for the whole patient is increasing. The need to evaluate severity of illness at this level is generated by the requirement for providers to treat patients with a number of diagnoses, as well as the need to plan and manage care within resource constraints.

This study described the application of severity of illness to inpatient data in the three hospitals of Syracuse, New York, which comprise a single, community wide acute care system. It was based on the application of All Patients Refined Diagnosis Related Group severity of illness system developed by $3 \mathrm{M}^{\mathrm{TM}}$ Health Information Systems to three indicators of utilization and outcomes. The study data were collected for indicators of efficiency and outcomes during the same twelve month period, January-December 2014.

The study demonstrated that the quantities of each of the three indicators included in the system increased with rising severity of illness. For adult medicine and adult surgery inpatient lengths of stay, medical-surgical inpatient readmission rates within 30 days, and post admission complication rates, levels increased from Minor to Moderate, Moderate to Major, and Major to Extreme severity of illness. It was interesting that these progressions occurred, especially for Potentially Preventable Readmissions and Potentially Preventable Complications that were developed in 2006 and 2007, more than ten years after the creation of the severity of illness algorithm.

These data suggested that severity of illness, at least in this version, is a valid tool for evaluating some forms of hospital utilization and outcomes. The fact that the analysis was carried out using administrative data indicated that the approach can be available widespread to providers and purchasers of care.

Perhaps more importantly, the study data also demonstrated that most problematic hospital stays, readmission rates, and complications in the Syracuse hospitals were focused in the populations with the highest severity of illness. Patients with Major and Extreme severity of illness were the source of obstacles to improving efficiency 
and outcomes identified in the data.

The study data indicated that most excess patient days in the Syracuse hospitals were generated by patients with high severity of illness. The data also indicated that these patients were associated with increased risk of inpatient readmissions and post admission complications.

The study suggested that the use of these data can contribute to the improvement of population health by challenging providers to improve care for patients at all levels of severity of illness. The sharing of information concerning these efforts could help improve population health on a local, regional, and national basis.

\section{Conclusions}

This information suggests that health care providers and payers will need to focus resources on management of populations by severity of illness as they work to improve the efficiency and effectiveness of care. It also suggests that they may be able to do so because many of the concerns that they need to address are related to these populations.

To be sure, the treatment and management of patients with high severity of illness are not an easy task. It requires addressing multiple diagnoses, as well as other clinical and demographic factors simultaneously. This challenge will require some of the highest creativity and related efforts that health care providers have to offer.

This area of care should generate useful directions for health systems at the community level. It addresses the need for effective care delivered in an efficient manner, especially for those patients who have the greatest needs. Understanding and managing by severity of illness hold the potential for raising the bar for this population and all of health care. Using severity of illness data can help providers and payers to reach these objectives.

\section{References}

[1] Dentzler, S. (2011) Urgent Measures for an Old Problem. Health Affairs, 30, 1626. http://dx.doi.org/10.1377/hlthaff.2011.0961

[2] Kellermann, A.D.L. (2011) A Decade of Health Care Cost Growth has Wiped out Real Income Gains for an Average US Family. Health Affairs, 30, 1630-1636. http://dx.doi.org/10.1377/hlthaff.2011.0585

[3] Skinner, J., Chandra, A., Goodman, D. and Fisher, E.S. (2009) The Elusive Connection between Health Care Spending and Quality. Health Affairs, 28, 1256-1258. http://dx.doi.org/10.1377/hlthaff.28.1.w119

[4] Zegers, M., de Bruine, M.C., Wagner, C., Hoonhout, L.H.F., Waaijman, R. and Smits, M. (2009) Adverse Events and Potentially Preventable Events in Dutch Hospitals: Results of a Retrospective Review Study. Quality in Health Care, 18, 297-302. http://dx.doi.org/10.1136/qshc.2007.025924

[5] Rau, J. (2012) Medicare to Penalize 2211 Hospitals for Excess Readmissions. Kaiser Health News.

[6] Fuller, R.L., McCullough, E.C., Bao, M.Z. and Averill, R.F. (2009) Estimating the Costs of Potentially Preventable Complications. Health Care Financing Review, 30, 17-32.

[7] Weil, A.R. (2015) Hospital Costs and Quality. Health Affairs, 34, 1263. http://dx.doi.org/10.1377/hlthaff.2015.0786

[8] Gawande, A.A. (2011) The Hot Spotters: Can We Lower Costs by Giving the Neediest Patients Better Care? New Yorker, January 24.

[9] Hasselman, D. (2013) Super Utilizer Summit: Common Themes for Innovative Complex Care Management. Princeton, Center for Health Care Strategies, New Jersey.

[10] Hong, C.S., Siegel, A.L. and Ferris, T.G. (2014) Caring for High Need, High Cost Patients: What Makes for a Successful Care Management Program? Commonwealth Fund, New York.

[11] Johnson, T.L., Rinehart, D.J., Durfee, J., Brewer, D., Batal, H., Blum, J., Oronce, C.J. and Melinkovich, P. (2015) For Many Patients Who Use Large Amounts of Health Care Services: The Need Is Intense Yet Temporary. Health Affairs, 34, 1312-1323. http://dx.doi.org/10.1377/hlthaff.2014.1186

[12] Fisher, E.S., Shortell, S.M., Kriendler, S.A., Van Citters, A.D. and Larson, B.K. (2012) A Framework for Evaluating the Formation and Implementation of Accountable Care Organizations. Health Affairs, 31, 2368-2378. http://dx.doi.org/10.1377/hlthaff.2012.0544

[13] 3M Health information Systems (1998) All Patients Refined Diagnosis Related Groups (APR-DRGs). 3M Health information Systems, Wallingford.

[14] 3M Health Information Systems (2008) Potentially Preventable Readmissions Classification System. 3M Health Information Systems, Wallingford.

[15] Hughes, J.S., Averill, R.F. and Goldfield, N.I. (2006) Identifying Potentially Preventable Complications Using a 
Present on Admissions Indicator. Health Care Financing Review, 27, 63-82.

[16] Lagoe, R.J., Pasinski, T., Kronenberg, P., Quinn, T. and Schaengold, P. (2006) Linking Health Services at the Community Level. Canada Healthcare Quarterly, 9, 60-65. http://dx.doi.org/10.12927/hcq..18229

[17] Lagoe, R.J., Nanno, D.S. and Luziani, M.E. (2012) Quantitative Tools for Addressing Hospital Readmissions. BMC Research Notes, 5, 620. http://dx.doi.org/10.1186/1756-0500-5-620

[18] Goldfield, N.I., McCullough, E.C., Hughes, J.S., Tang, A.M., Eastman, B., Rawlins, L.K. and Averill, R.F. (2008) Identifying Potentially Preventable Readmissions. Health Care Financing Review, 30, 75-92. 